\title{
Post acuerdo de paz: Una etapa a legitimar bajo el calediscopio de las Mipymes colombianas
}

\section{Post-peace agreement: A stage to be legitimized under the calediscope of the Colombian Mipymes}

\author{
DOI: https://doi.org/10.17981/juridcuc.16.1.2020.13
}

Fecha de Recepción: 23/03/2020 Fecha de Aceptación: 15/04/2020

\author{
Rafael Ravina-Ripoll \\ Universidad de Cádiz. Cádiz (España) \\ rafael.ravina@uca.es
}

\author{
Edgar Julián Gálvez-Albarracín \\ Universidad del Valle. Cali (Colombia) \\ edgar.galvez@correounivalle.edu.co
}

\author{
Bairon Otálvaro-Marín \\ Universidad del Valle. Cali (Colombia) \\ bairon.otalvaro@correounivalle.edu.co
}

Para citar este artículo:

Ravina-Ripoll, R., Gálvez-Albarracín, E. \& Otálvaro-Marín, B. (2020). Post acuerdo de paz: Una etapa a legitimar bajo el calediscopio de las Mipymes colombianas. Jurídicas CUC, 16(1), 303-322. DOI: http://dx.doi.org/10.17981/juridcuc.16.1.2020.13

\section{Resumen}

El conflicto armado colombiano es reconocido mundialmente por ser uno de los más antiguos del planeta y por haber intentado diferentes iniciativas para solucionarlo; en relación con ello este trabajo empírico y transversal aporta nueva información sobre la intención de los empresarios nacionales por participar en acciones económicas destinadas a legitimar jurídicamente la construcción de paz. Los resultados obtenidos mediante una muestra de 482 micro, pequeñas y medianas empresas (Mipymes) del país indican que existe mayor propensión por apoyar a las víctimas que a los excombatientes en cuanto a generarles empleo y vincularse a sus emprendimientos, siendo notoriamente baja la disposición de contribuir a conformar un fondo para financiar actividades para consolidar el post acuerdo. Se concluye que es necesario evitar la polarización, y sensibilizar más a los gerentes y empresarios sobre la importancia de su rol para construir una estabilidad social y política duradera. Teniendo presente que la cultura de la paz juega un papel muy relevante en el progreso económico y democrático de Colombia en el mundo globalizado.

Palabras clave: Mipymes; construcción de paz; post acuerdo; desarrollo sostenible

\section{Abstract}

The Colombian armed conflict is recognized worldwide for being one of the oldest on the planet and for having tried different initiatives to solve it; in relation to this, this empirical and transversal work provides new information on the intention of national businessmen to participate in economic actions aimed at legally legitimizing peace building. The results obtained through a sample of 482 micro, small and medium enterprises (Mipymes) in the country indicate that there is a greater propensity to support the victims than the ex-combatants in terms of generating employment for them and linking them to their enterprises, with a notoriously low willingness to contribute to a fund to finance activities to consolidate the postagreement. The conclusion is that it is necessary to avoid polarization, and to make managers and businessmen more aware of the importance of their role in building lasting social and political stability. Bearing in mind that the culture of peace plays a very relevant role in the economic and democratic progress of Colombia in the globalized world.

Keywords: Mipymes; peace building; post agreement; sustainable development 


\section{INTRODUCCIÓN}

Las diferentes propuestas y procesos de paz vividos en Colombia desde el periodo presidencial de Belisario Betancur (1982-1986) hasta el de Juan Manuel Santos (2012-2018), reflejan, al menos aparentemente, el interés de los actores vinculados con la guerra por abandonar la lucha armada y buscar una solución política negociada al conflicto interno (González, 2014), además del cansancio de la sociedad colombiana frente a la violencia sufrida en el territorio nacional.

El máximo logro en la coyuntura política reciente es el acuerdo de paz entre la guerrilla de las Fuerzas Revolucionarias de Colombia (FARC-EP) y el gobierno colombiano, el cual se firmó en Bogotá el 24 de noviembre del 2016, dando inicio a una serie de desafíos políticos, sociales, institucionales, económicos y culturales relacionados con: a) La reforma rural integral del campo colombiano, b) la apertura democrática para la consolidación de la paz, c) la reincorporación efectiva de los excombatientes a la vida comunitaria, d) la búsqueda de soluciones al problema de las drogas ilícitas, e) el fortalecimiento de un sistema integral de verdad, justicia, reparación y no repetición, y f) la verificación, seguimiento y evaluación del acuerdo final de paz por parte de un actor internacional (Estrada, 2019).

El estado de avance de los procesos de implementación temprana de los acuerdos de paz en Colombia durante lo corrido del gobierno del presidente Iván Duque Márquez (2018-2022), muestran una modalidad sofisticada de incumplimiento mediante una reinterpretación de lo acordado durante la formulación e implementación del Plan Nacional de Desarrollo "Pacto por Colombia, pacto por la Equidad" (Departamento Nacional de Planeación, DNP, 2019). La política de paz con legalidad desconoce aspectos centrales de los acuerdos pactados, en particular el enfoque territorial, al tiempo que inactiva la arquitectura institucional en la reincorporación socioeconómica de los excombatientes (Comisión de Seguimiento, Impulso y Verificación a la Implementación del Acuerdo Final-CSIVI, FARC, Centro de Pensamiento y Diálogo Político, 2019). 
Uno de los enfoques más desarrollados por la academia colombiana para analizar los retos del post acuerdo, es la efectiva construcción de la cultura de la paz, es decir, cultivar un ecosistema legislativo donde brote la igualdad de oportunidades, el respeto a los derechos humanos y los principios de responsabilidad empresarial del ordenamiento jurídico colombiano (Hernández, Chumaceiro, Ziritt y Acurero, 2018; Hernández, Meléndez, Chumaceiro y Gil, 2017; Ibarra-Padilla, 2014). Esto precisa, entre otras cosas, de la participación activa de los diferentes actores de la sociedad, incluidas las empresas como principales generadores de empleo, riqueza y desarrollo económico nacional y regional (Rettberg, 2008). En este sentido los empresarios pueden involucrarse en dichas iniciativas, por ejemplo, incidiendo políticamente para el logro de reformas jurídicas en el Estado, así como aportando recursos monetarios para aliviar los daños sufridos por las infraestructuras civiles durante la guerra (Arteaga et al., 2012).

Es importante mencionar también que en el país se han desarrollado diferentes iniciativas para apoyar la construcción de paz tanto fuera como dentro de las empresas, es el caso del proyecto "Construcción de paz desde Confecámaras y las Cámaras de Comercio de Colombia" el cual fue elaborado gracias a una alianza entre la Fundación Ideas para la Paz, Confecámaras ${ }^{1}$ (Confederación de Cámaras de Colombia) y la Cámara de Comercio de Bogotá, buscando proponer una hoja de ruta estratégica para que dichas organizaciones fortalezcan su rol como dinamizadoras de la reconciliación y la convivencia nacional. De esta manera, las Mipymes podrá cumplir con los principios normativos de la Constitución del año 1991, donde se declara, entre otras cosas, a Colombia como un Estado Social de Derecho.

Unido a lo anterior, se debe indicar que las empresas colombianas, -según cifras del Registro Único Empresarial y Social (RUES) coordinando las cámaras de comercio, y el Departamento Nacional de Estadísticas (DANE)-, las Micro, Pequeñas y Medianas (Mipymes)

${ }^{1}$ Confecámaras, es una entidad privada, sin ánimo de lucro, que agremia y representa a las 57 entidades del sector que existen en Colombia. 
constituyen la mayor parte de su demografía, aportando el $67 \%$ del empleo y el 28\% del producto interno bruto nacional (dinero. com, 2016). Esto les hace ser unos agentes de gran relevancia para el desarrollo de la economía y para la construcción del Estado de Bienestar (Aguilera, Ávila, y Solano, 2017).

Por último cabe mencionar que si bien en el país se han llevado a cabo estudios como la Encuesta Empresarial de Paz de la Cámara de Comercio de Bogotá, en el cual se consulta a los empresarios sobre temas como su percepción de los procesos de negociación con las guerrillas, la justicia transicional y su disposición de participar en acciones e iniciativa económicas para facilitar la reconciliación nacional, los resultados no se discriminan según el tamaño de las empresas, por lo que no se puede conocer específicamente la posición del sector Mipyme, el cual por la relevancia previamente señalada juega un papel trascendental en la consolidación de estabilidad económica y social y en general del desarrollo sostenible de Colombia.

\section{Desarrollo}

\section{MARCO TEÓRICO Y ESTUDIOS PREVIOS}

Existen diversos retos asociados a promover una cultura de paz en sociedades que han vivido prolongados periodos de guerra, y a la búsqueda de relaciones de convivencia que permitan la transformación de las problemáticas de exclusión y desigualdad (Uribe, 2018). En dicho sentido en este apartado se reflexiona sobre la construcción de paz y la vinculación de la actividad empresarial como alternativa de desarrollo económico y jurídico para la consolidación de una sociedad democrática y equitativa como lo requiere Colombia.

La implementación del acuerdo final con las FARC-EP para la terminación del conflicto y la construcción de una paz estable y duradera en Colombia, avanza de forma parcial y con baja intensidad, observándose en la coyuntura actual la reaparición o recrudecimiento de muy diversas formas de violencia rural y urbana, a tal punto que podrían impedir su continuidad y consolidación (Plataforma Colombiana de Derechos Humanos, Democracia y Desarrollo- 
PCDHDD, Coordinación Colombia Europa Estados Unidos-CCEEU y Alianza de Organizaciones Sociales y Afines, 2019).

El logro de una sociedad con transformaciones democráticas que legitimen la participación, la equidad y la convivencia pacífica de los colombianos, implica el compromiso decidido de los diversos actores del mercado y del Estado. Así se podrá mitigar las causas del conflicto violento vivido, así como desarrollar procesos basados en la consolidación de un bienestar subjetivo para la población (RavinaRipoll, Tobar-Pesantez y Marchena-Domínguez, 2019; Otálvaro, 2017).

La comprensión de los alcances sociales y económicos de los acuerdos de paz con la Guerrilla de las FARC-EP se pueden establecer en una perspectiva histórica, sistémica, jurídica y territorial (Estrada, 2019). Desde una perspectiva histórica, es uno de los momentos más importantes de la coyuntura política e institucional del país en el siglo $\mathrm{XXI}$, en cuanto a lo sistémico incorpora un conjunto de disposiciones y acciones necesarias para desarrollar un proceso normativo orientado a la terminación del conflicto y la búsqueda de una paz estable y duradera. Y en cuanto a lo territorial, se plantearon una serie de acciones con enfoque territorial que buscan el cese de agresiones militares y el bienestar colectivo en el ámbito local, especialmente en las comunidades campesinas, indígenas y afrodescendientes. Como es sabido estos grupos sociales han vivido con mayor intensidad el conflicto armado en sus territorios (Estrada, 2019).

El marco teórico referencial de este trabajo se circunscribe a la construcción de paz, proceso que se prevé complejo al reconocer las tramas implicadas en la solución del conflicto armado colombiano a nivel legal y territorial (Muñoz, 2001). En dicho sentido se destaca la teoría propuesta por el autor citado, que se orienta a comprender la "paz imperfecta", que centra su mirada en la coexistencia de procesos de gestión de los conflictos de manera pacífica y violenta simultáneamente (Muñoz y López, 2001).

Colombia es un territorio en donde convive la guerra y la paz, es un contexto que tiene una variedad actores institucionales, sociales, económicos, internacionales, comunitarios, legales e ilegales que 
coexisten en la profundización del conflicto o en la búsqueda de una paz estable y duradera que propicie una ruptura frente a las dinámicas de violencia habitual (Moreno, 2014). Los principales desafíos en esta perspectiva se orientan a resolver los siguientes problemas estructurales: a) El aumento de relaciones sociales de exclusión y desigualdad presentes en los territorios, b) la búsqueda de caminos para avanzar en la construcción de justicia social y paz de carácter universal, c) la promoción de experiencias y estrategias de movilización social por la paz que intenten desde lo cotidiano transformar relaciones desiguales e inequitativas.

La investigación y construcción de un marco teórico para la paz es un campo reciente, impulsado desde las ciencias jurídicas y desde diversos campos transdisciplinares, que está indagando por el pasado, el presente y el futuro de sociedades interesadas en la construcción de paz (Galtung, 1995, p. 347). En Colombia se ha centrado la mirada en los estudios de la violencia y la guerra, quizás esto pueda ser un obstáculo para la búsqueda de alternativas orientadas a la construcción de la paz, especialmente en los territorios; o como dice Fisas (2004, p. 41) "hemos acumulado una gran experiencia para hacer la guerra y muy poca sabiduría para hacer la paz", o "valorando la paz hemos pensando más en clave de violencia" (Muñoz, 2001, p. 23).

Colombia en el marco de lo que se ha denominado una "paz estable y duradera" viene construyendo una serie de reflexiones sobre el avance de los procesos de implementación de los acuerdos logrados con la guerrilla de las FARC-EP, miradas que brindan variedad de posibilidades de estudiarla desde tres grandes tendencias teóricas: i) Paz negativa, ii) paz positiva, iii) paz imperfecta (Moreno, 2014). La paz negativa o la ausencia de cualquier tipo de violencia, surge ante la necesidad de encontrar un equivalente moral y científico a la guerra, para ponerle fin a los desastres generados en la Primera Guerra Mundial, es concebida como la ausencia de guerra o de violencia.

La paz positiva que evita o reduce todas las expresiones de violencia, ofrece la posibilidad de indagar sobre la paz y su relación con la justicia, "generadora de valores positivos y perdurables, capaz de integrar política y sociedad, generar expectativas, y contemplar la 
satisfacción de las necesidades humanas" (Muñoz, 2001, p. 4). Esta paz se relaciona con la estructura y las formas "ocultas y estáticas de la violencia, y sus sistemas de miseria, dependencia, hambre, desigualdades de género, etc." (Muñoz, 2001, p. 4). La paz imperfecta o de construcción de paz en medio del ciclo de la violencia como sucede en Colombia (Moreno, 2014), permite reconocer aspectos fundamentales de la existencia humana, entre ellos la idea de que el conflicto es inherente a las sociedades, y que por tanto se requieren mecanismos de mediación de la violencia o mediación de la no violencia.

A nivel nacional se han desarrollado trabajos para conocer y potenciar la forma en que las empresas se están vinculando a la construcción de una paz estable y duradera; es el caso del proyecto "Construcción de paz desde Confecámaras y las Cámaras de Comercio de Colombia”, en el cual se revisó cómo estas entidades lo hacen desde las siguientes áreas estratégicas: Fortalecimiento empresarial, impulso de las agendas de competitividad, participación ciudadana, mecanismos alternativos de solución de conflictos y alianzas estratégicas con actores públicos, privados y sociales. ¿Cómo puede el sector empresarial aportar a la paz?, se puede responder de la siguiente manera (Fundación Ideas para la Paz, 2017):

Una empresa u otro actor del sector privado, como las Cámaras de Comercio, desde su gestión y por medio de acciones concretas, tiene un impacto positivo en la construcción de la paz duradera en país cuando, con un enfoque de acción sin daño, logra aportar a la creación de condiciones socioeconómicas, ambientales, políticas y socioculturales pacíficas, justas e incluyentes, así como al establecimiento de relaciones de confianza, reconciliación y solución no-violenta de diferencias entre personas, grupos y otros actores sociales, privados y públicos (p. 10).

En resumen, es importante entender las cuestiones simbólicas, legales y materiales en el análisis de la paz, como procesos inacabados, pero que poseen una potencia transformadora para avanzar hacia la construcción de paz territorial (Jiménez, 2016). Estas acciones implican una permanente construcción y en el cual los empresarios y gerentes tienen un importante rol a desempeñar. 


\section{Metodología}

La población objeto esta conformada por las Mipymes inscritas en el registro mercantil de las cámaras de comercio de las cuatro principales ciudades de Colombia: Barranquilla, Bogotá, Cali y Medellín. Los datos se recogieron telefónicamente entre julio y noviembre del año 2017 mediante un cuestionario que tomó como referencia el aplicado en la Encuesta Empresarial de Paz de la Cámara de Comercio de Bogotá, y fue administrado a los propietarios y/o gerentes de las organizaciones abordadas por parte de una agencia de investigación de mercados contratada para tal efecto.

La Tabla 1 indica que la muestra esta conformada por 482 empresas, 91 de ellas ubicadas en Medellín (18,8\%), 183 en Cali (38,0\%) y 208 en Bogotá (43,2\%), las Mipymes de Barranquilla no contestaron la encuesta a pesar de su importancia, constituyéndose en una limitación de la investigación y así mismo se aprecia como el 17,6\% de las organizaciones abordadas son micro, $47,7 \%$ pequeñas y el restante $34,6 \%$ medianas.

TABLA 1.

Distribución de la muestra por ubicación y tamaño de las empresas

\begin{tabular}{llllll}
\hline \multicolumn{2}{c}{ Distribución geográfica } & \multicolumn{2}{c}{ Distribución por tamaño de empresas } \\
\multicolumn{1}{c}{ Ciudad } & Cantidad & Porcentaje & Tamaño & Cantidad & Porcentaje \\
\hline Bogotá. D. C. & 208 & $43,2 \%$ & Micro & 85 & $17,6 \%$ \\
Cali & 183 & $38,0 \%$ & Pequeñas & 230 & $47,7 \%$ \\
Medellín & 91 & $18,8 \%$ & Medianas & 167 & $34,6 \%$ \\
\hline Totales & 482 & $100 \%$ & Totales & 482 & $100 \%$ \\
\hline
\end{tabular}

Fuente: Elaboración propia a partir de los datos recolectados en la investigación.

Por su parte la Tabla 2 señala que la mayor parte de las empresas de la muestra están ubicadas en el sector servicios (48,3\%), 21,2\% en el industrial, 19,7\% en el comercial, 7,1\% en la agricultura o ganadería y $3,7 \%$ en la construcción; de igual manera que el 70,3\% de ellas son de naturaleza familiar (es decir que su propiedad y administración es de una o más familias), y el 29,7\% son organizaciones no familiares. 
TABLA 2.

Distribución de la muestra por sector de actividad y tipo de propiedad de las empresas

\begin{tabular}{llllll}
\hline \multicolumn{2}{c}{ Distribución por sector de actividad } & \multicolumn{3}{c}{ Distribución según tipo de propiedad } \\
\multicolumn{1}{c}{ Sector } & Cantidad & Porcentaje & ¿Familiar? & Cantidad & Porcentaje \\
\hline Industria & 102 & $21,2 \%$ & & & \\
Comercio & 95 & $19,7 \%$ & & 339 & $70,3 \%$ \\
Servicios & 233 & $48,3 \%$ & $\mathrm{Si}$ & 143 & $29,7 \%$ \\
Construcción & 18 & $3,7 \%$ & No & & \\
$\begin{array}{l}\text { Agricultura o } \\
\text { ganadería }\end{array}$ & 34 & $7,1 \%$ & & & \\
\hline Totales & 482 & $100 \%$ & & 482 & $100 \%$ \\
\hline
\end{tabular}

Fuente: Elaboración propia a partir de los datos recolectados en la investigación.

El instrumento con que se midió el fenómeno analizado se denominó Intención de Participar en Actividades del Pos acuerdo (IPAP), el cual se puede observar en la Tabla 3 y está conformado por 5 prácticas sobre las cuales se consultó a los empresarios/gerentes a través de una escala Likert de 7 posiciones, de tal manera que se pudiera conocer tanto el nivel de intención respecto a cada una, como el global (o total IPAP), el cual se establece mediante el promedio de cada práctica.

TABLA 3.

Intención de participar en actividades del pos acuerdo (IPAP)

Por favor califique el nivel de probabilidad de que su empresa participe en cada una de las siguientes actividades del "pos acuerdo", utilice una escala de 1 a 7 donde 1: es imposible, 2: poco posible, 3: medianamente posible, 4: indeciso, 5: probablemente, 6: muy probablemente, y 7: con toda seguridad.

Crear empleo para excombatientes.

Crear empleo para víctimas.

Apoyar emprendimientos de excombatientes.

Apoyar emprendimiento de víctimas.

Aportar dinero a un fondo para la paz en el país.

Total IPAP

Fuente: Adaptado de la Cámara de Comercio de Bogotá (2015). 


\section{RESULTADOS Y DISCUSIÓN}

A continuación se presentan y discuten los resultados obtenidos en la investigación, comenzando con los generales, es decir los de todas las empresas abordadas, y discriminándolos luego grupalmente según las características de las organizaciones tenidas en cuenta.

En primer lugar la Tabla 4 muestra que el total IPAP para el grupo de empresas fue de 4,50 el cual está en el rango entre la indecisión y la probabilidad de participación, esto sugiere que hay una razonable esperanza de que las Mipymes colombianas apoyen iniciativas empresariales como las consultadas en favor de la consolidación del pos acuerdo, lo cual es una de las bases de lo que el gobierno nacional ha denominado Planes de Desarrollo con Enfoque Territorial (PDET) y de las estrategias pro recuperación de las Zonas más Afectadas con el Conflicto Interno (ZOMAC) (Cámara de Comercio de Bogotá, Consejo Gremial Nacional, Centro de Investigaciones y Proyectos Especiales-CIPE, 2019).

En cuanto a las prácticas o acciones específicas la misma Tabla 4 muestra que hay acciones que definitivamente no serían implementadas por ninguna de las empresas (ya que su mínimo general es 1 sobre 7), pero que hay otras que con alta probabilidad las adoptarían casi todas las Mipymes (ya que el máximo individual es de 7 y el general de 6,60); es importante notar que las que tiene mayores probabilidades de adopción son las relacionadas con las víctimas y con los emprendimientos respectivamente, así primero está apoyar emprendimientos de víctimas (5.06), seguido de crear empleo para víctimas (4,79); luego apoyar emprendimientos de excombatientes $(4,47)$ y crear empleo para excombatientes $(4,44)$.

Estos resultados confirman los hallazgos de la "Encuesta empresarial nacional de paz" de la Cámara de Comercio de Bogotá (2018) en donde sólo el $34 \%$ de los encuestados se mostraron inclinados a contratar excombatientes, frente a un 39\% que apoyaría sus emprendimientos, y la "Encuesta empresarial sobre construcción de pazBogotá" (Cámara de comercio de Bogotá, 2015) donde también se encuentra mejor predisposición hacia las victimas que hacia los excombatientes. 
De igual manera la Tabla 4 deja ver que la acción que tiene peor recibo es aportar dinero a un fondo para la paz (3,73 sobre 7), lo cual muestra iguales resultados en la "Encuesta empresarial sobre construcción de paz-Bogotá" del 2015, en donde sólo el 65\% de los empresarios estuvieron dispuestos a participar. Estos resultados podrían demostrar como los empresarios consideran que el pago normal de impuestos realizado se contribuye suficientemente para el desarrollo social, pero también demuestra la inconformidad con los acuerdos, y/o la desconfíanza en el manejo que se podría dar a los aportes adicionales.

TABLA 4.

Intención participar en actividades del pos acuerdo (IPAP)

\begin{tabular}{lllll}
\hline \multicolumn{1}{c}{ Prácticas } & Mínimo & Máximo & Media & $\begin{array}{c}\text { Desviación } \\
\text { Estándar }\end{array}$ \\
\hline $\begin{array}{l}\text { Crear empleo para } \\
\text { excombatientes }\end{array}$ & 1 & 7 & 4,44 & 1,19 \\
$\begin{array}{l}\text { Crear empleo para } \\
\text { víctimas }\end{array}$ & 1 & 7 & 4,79 & 1,71 \\
$\begin{array}{l}\text { Apoyar emprendimientos } \\
\text { de excombatientes }\end{array}$ & 1 & 7 & 4,47 & 1,56 \\
$\begin{array}{l}\text { Apoyar emprendimiento } \\
\text { de víctimas }\end{array}$ & 1 & 7 & 5,06 & 1,69 \\
$\begin{array}{l}\text { Aportar dinero a un fondo } \\
\text { para la paz en el país }\end{array}$ & 1 & 7 & 3,73 & 1,46 \\
Total IPAP & 1 & 6,60 & 4,50 & 1,00 \\
\hline
\end{tabular}

Fuente: Elaboración propia a partir de los datos recolectados en la investigación.

En seguida se presentan los resultados discriminados según las características empresariales consideradas para el estudio, en primer lugar y respecto a la ubicación geográfica de las Mipymes puede apreciarse en la Tabla 5 que en la ciudad donde hay mayor intención de apoyar globalmente las iniciativas consultadas es en Medellín $(4,90)$, seguida de Bogotá $(4,60)$ y por último en Cali $(4,18)$. Estos hallazgos podrían tener relación con la tradicional "cultura paisa" reinante en la primera ciudad y que se reconoce nacionalmente por su laboriosidad, solidaridad y generosidad. También se observa en 
la misma Tabla que en todas las ciudades la alternativa que tendría mayor aceptación es apoyar el emprendimiento de víctimas (Medellín 5,55 , Bogotá 5,17 y Cali 4,69) y el que menos aportar dinero para un fondo para la paz (Cali 3,25, Bogotá 3.96 y Medellín 4,19).

En relación con las diferencias según el tamaño de las empresas, la misma Tabla 5 muestra como a medida que la empresa es más grande el total IPAP aumenta (medianas 4,60, pequeñas 4,55 y micros 4,16), estos resultados podrían señalar por una parte que en las empresas de mayor tamaño se sienten con mayores recursos y capacidades para vincularse con iniciativas como las señaladas, y/o que se tiene mayor conciencia de la importancia de contribuir a la paz de la región y el país. En cuanto al comportamiento de cada una de las alternativas consultadas, se observa que es similar al general, es decir que no importa el tamaño hay mayor disposición para apoyar a las víctimas que a los excombatientes, y en último lugar para hacer aportes económicos a un fondo.

TABLA 5.

Intención participar en actividades del pos acuerdo (IPAP) según ubicación y tamaño de las empresas

\begin{tabular}{lcccccc}
\hline \multicolumn{1}{c}{ Prácticas } & \multicolumn{3}{c}{ Ubicación de la empresa } & \multicolumn{4}{c}{ Tamaño de la empresa } \\
& Bogotá & Cali & Medellín & Micro & Pequeñas & Medianas \\
\hline $\begin{array}{l}\text { Crear } \\
\text { empleo para } \\
\text { excombatientes }\end{array}$ & 4,41 & 4,30 & 4,78 & 4,18 & 4,55 & 4,43 \\
$\begin{array}{l}\text { Crear empleo } \\
\text { para víctimas }\end{array}$ & 5,12 & 4,27 & 5,09 & 4,18 & 4,86 & 5,01 \\
$\begin{array}{l}\text { Apoyar } \\
\text { emprendimientos } \\
\text { de excombatientes }\end{array}$ & 4,34 & 4,40 & 4,88 & 4,26 & 4,57 & 4,43 \\
$\begin{array}{l}\text { Apoyar } \\
\text { emprendimiento } \\
\text { de víctimas }\end{array}$ & 5,17 & 4,69 & 5,55 & 4,85 & 5,05 & 5,19 \\
$\begin{array}{l}\text { Aportar dinero a } \\
\text { un fondo para la } \\
\text { paz en el país }\end{array}$ & 3,96 & 3,25 & 4,19 & 3,35 & 3,73 & 3,94 \\
\hline \begin{tabular}{l} 
Total IPAP \\
\hline
\end{tabular} & 4,60 & 4,18 & 4,90 & 4,16 & 4,55 & 4,60 \\
\hline
\end{tabular}

Fuente: Elaboración propia a partir de los datos recolectados en la investigación. 
En cuanto a las particularidades de la IPAP general según el sector de actividad de las empresas, puede apreciarse en la Tabla 6 que es en la construcción donde más alta se encuentra $(4,60)$, seguido de la industria $(4,55)$, el comercio $(4,54)$, los servicios $(4,47)$ y por último en el sector agricultura y la ganadería $(4,36)$. Estos resultados podrían deberse a que en el primer sector es común que se vinculen como obreros a personas de bajo nivel social, donde precisamente se ubican muchos de quienes se han visto implicadas en el conflicto armado; sin embargo también es contradictorio que sea en las empresas de agricultura y ganadería donde menos IPAP haya, pues es históricamente se ha visto envuelto perjudicado por la problemática asociada a la violencia.

Continuando con el análisis sectorial se observa que donde hay mayor propensión para crear empleo para excombatientes es en la agricultura y la ganadería $(4,53)$ y donde menos en los servicios y en la construcción (ambos con 4,39), donde más se haría para victimas es en la construcción $(5,00)$ y donde menos en la agricultura y la ganadería $(4,44)$. Donde más se apoyaría emprendimientos de excombatientes es en la industria $(4,57)$ y donde menos en la agricultura y ganadería $(4,18)$, donde más se apoyarían los de las victimas sería en la construcción $(5,61)$ y donde menos en la agricultura y ganadería $(4,88)$; por último donde mayor disposición se haya de aportar a un fondo para la paz es en la industria $(3,82)$ y donde menos en el comercio $(3,64)$.

Por otra parte en la misma Tabla 6 se ve que en las empresas de propiedad familiar hay una levemente mayor IPAP $(4,53)$ que en las que no lo son $(4,48)$, esto se podría explicar desde la presunción de que en las primeras se presenta a nivel global una mayor propensión a la solidaridad social, como extensión de la común a nivel familiar. De igual manera es importante notar que el apoyo para los excombatientes es más alto en el caso de las empresas no familiares, 4,51 frente a 4,28 para el caso de la generación de empleo, y 4,50 frente a 4,38 para el de los emprendimientos, lo cual se explicaría en que los empresarios familiares precisamente por proteger a sus allegados son menos propensos a dar oportunidades a los antiguos combatientes. 
TABLA 6.

Intención participar en actividades del posconflicto (IPAP) según sector de actividad y tipo de propiedad de las empresas

\begin{tabular}{|c|c|c|c|c|c|c|c|}
\hline \multirow{2}{*}{ Prácticas } & \multicolumn{5}{|c|}{ Sector de actividad } & \multicolumn{2}{|c|}{ ¿Familiar? } \\
\hline & Ind & Comerc & Serv & Const & Agric o Gan & $\mathrm{Si}$ & No \\
\hline $\begin{array}{l}\text { Crear } \\
\text { empleo para } \\
\text { excombatientes }\end{array}$ & 4,47 & 4,52 & 4,39 & 4,39 & 4,53 & 4,28 & 4,51 \\
\hline $\begin{array}{l}\text { Crear empleo } \\
\text { para víctimas }\end{array}$ & 4,70 & 4,92 & 4,82 & 5,00 & 4,44 & 5,02 & 4,69 \\
\hline $\begin{array}{l}\text { Apoyar } \\
\text { emprendimientos } \\
\text { de } \\
\text { excombatientes }\end{array}$ & 4,57 & 4,51 & 4,46 & 4,33 & 4,18 & 4,38 & 4,50 \\
\hline $\begin{array}{l}\text { Apoyar } \\
\text { emprendimiento } \\
\text { de víctimas }\end{array}$ & 5,21 & 5,13 & 4,96 & 5,61 & 4,88 & 5,20 & 5,00 \\
\hline $\begin{array}{l}\text { Aportar dinero a } \\
\text { un fondo para la } \\
\text { paz en el país }\end{array}$ & 3,82 & 3,64 & 3,73 & 3,67 & 3,76 & 3,78 & 3,71 \\
\hline Total IPAP & 4,55 & 4,54 & 4,47 & 4,60 & 4,36 & 4,53 & 4,48 \\
\hline
\end{tabular}

Fuente: Elaboración propia a partir de los datos recolectados en la investigación.

\section{Conclusiones}

Grasa, Carvajalino y Duque (2019) señalan que el proceso de construcción de paz en Colombia es una importante oportunidad para que los empresarios fortalezcan su vinculación al desarrollo del país. Para ello se hace preciso: La inclusión de la población afectada por el conflicto armado interno, la producción respetuosa del medio ambiente y la reconstrucción del tejido jurídico y social. Esto no será posible si no se implanta el concepto del "valor compartido". Por este término se entiende aquellas innovaciones en los negocios, que son rentables pero también generan beneficios sociales y ambientales, todo ello en sintonía con la Agenda 2030 de Desarrollo Sostenible (ODS). 
Los resultados de esta investigación muestran de manera general que los empresarios y gerentes Mipymes colombianos tienen una moderada inclinación positiva a implementar acciones que contribuyan en el marco de lo acordado con la guerrilla de las FARC-EP, a la construcción de una paz y al desarrollo sostenible, lo cual lógicamente es esperanzador. Sin embargo es innegable e inocultable también la existencia de una considerable proporción que no estaría dispuesta a ello. Tal hecho podría estar influido por la polarización que se ha vivido y ha sido liderada por la clase política, y en particular por la perteneciente al gobierno nacional actual, que desde el proceso de negociaciones con las FARC-EP se mostró en desacuerdo con este, y como se señaló previamente ha venido tratando de cambiar la interpretación de lo acordado.

Por otra parte y como era de esperarse, los hallazgos confirman que los empresarios tienen más propensión a apoyar a las víctimas que a los excombatientes. Esto es entendible, pero refuerza la necesidad de que reflexionen sobre las razones por las cuales según los casos conocidos, muchas personas se vieron implicadas como actores activos del conflicto, ya sea por el reclutamiento obligado o como única opción de encontrar recursos económicos para enfrentar su exclusión social y laboral (Villarraga-Sarmiento, 2013).

Es importante entonces que toda la sociedad colombiana sea más consciente de su historia, y especialmente de una de las causas principales del conflicto armado en el país, la desigualdad económica, de la cual directa o indirectamente los empresarios han sido actores principales, ya sea como generadores o como mitigadores. En este sentido es importante que ellos y en general la población entienda como señala Muñoz (2001) es preferible una "paz imperfecta", que una confrontación perfecta.

A continuación se señalan primero las limitaciones y luego las futuras líneas de investigación que se pueden desprender del trabajo: 1) La muestra sólo cubre empresas de 3 ciudades, ya que como se dijo previamente los gerentes de Barranquilla no respondieron la encuesta; al respecto, futuros trabajos pueden 
abordar más ciudades, incluyendo no capitales y ojalá algunas ubicadas en las ZOMAC y con esto se tendrá una mayor representatividad nacional; y 2) no se haya consultado a los empresarios sobre estímulos que podrían impulsar su IPAP, tales como descuentos tributarios, cofinanciación gubernamental para crear nuevos empleos, etc.; próximas investigaciones serían útiles para confirmar la forma como ellos influirían en elevar los resultados obtenidos.

Los resultados de esta investigación tienen implicaciones para los gobiernos nacionales, regionales y locales, pues les indica como la radicalización que la clase política ha propiciado en la sociedad puede ser una de las causas de que el IPAP de los empresarios Mipyme de Colombia no sea mejor, además les señala las ciudades y las acciones que se requieren para un mayor estímulo financiero y de otro tipo, para fortalecer el compromiso empresarial con la construcción de paz y pueda ser más alto.

Igualmente a los empresarios y a sus gremios les muestra como se requiere un mayor compromiso de las Mipymes con la consolidación del pos acuerdo, lo cual seguramente redundará en un mejor clima de negocios y seguridad jurídica. Por último, a la academia le señala las cuestiones requeridas para mayor investigación, así mismo le muestra la necesidad de trabajar aún más en la formación de empresarios y gerentes comprometidos socialmente. Desde la perspectiva jurídica, este artículo ha detectado la necesidad de un construir un marco jurídico bajo la arquitectura de los valores éticos y el capitalismo humanista. Esto conlleva a que los máximos responsables de la Mipymes colombianas se comprometan a edificar su progreso empresarial a través de la búsqueda del buen vivir y las emociones positivas de sus ciudadanos en la era del pos acuerdo de la paz.

\section{REFERENCIAS}

Aguilera, A., Ávila, G. y Solano, O. (2017). Las TIC en la formulación estratégica de las Pymes de Santiago de Cali-Colombia. Entramado, 13(1), 111-112. 
Arteaga, B., Walteros, D., Puentes J., Salcedo, D., Andrade, O., Bautista, D., Parra, E., Vargas, D. y Baños, L. (2012). Empresa y construcción de paz. Cuadernos paz a la carta No. 3. Bogotá, D.C.: Editorial Universidad Jorge Tadeo Lozano. Recuperado de https://www.utadeo.edu.co/sites/tadeo/files/collections/documents/field_attached_file/ cuaderno3.pdf? width $=740 \&$ height $=780 \&$ inline $=$ true $\# p$ df_reader

Cámara de Comercio de Bogotá. (2018). Encuesta Empresarial Nacional de Paz. [Infografía]. Bogotá, D.C.: Centro de información empresarial (CIEB). Recuperado de: https:// bibliotecadigital.ccb.org.co/handle/11520/20341

Cámara de Comercio de Bogotá. (2015). Encuesta empresarial sobre construcción de paz - Bogotá. [Infografía]. Bogotá, D.C.: Centro de información empresarial (CIEB). Recuperado de https://bibliotecadigital.ccb.org.co/handle/11520/18743

Cámara de Comercio de Bogotá, Consejo Gremial Nacional, CIPE. (2019). Resultados del Observatorio de Inversión Privada en las zonas más afectadas por la violencia. [Presentación]. Bogotá, D.C.: JA\&A. Recuperado de http://hdl. handle.net/11520/24237

CSIVI, FARC, Centro de Pensamiento y Diálogo Político (2019). La implementación del Acuerdo de paz durante el gobierno de Iván Duque. Bogotá, D.C.: Impresol.

dinero.com (abril 14, 2016). Mipymes generan alrededor del $67 \%$ del empleo en Colombia. Dinero, Recuperado de https://www.dinero.com/edicion-impresa/pymes/articulo/ evolucion-y-situacion-actual-de-las-mipymes-en-colombia/222395

Estrada, J. (Coord.) (2019). El acuerdo de paz en Colombia. Entre la perfidia y la potencia transformadora: Entre la perfidia y la potencia transformadora. Bogotá, D.C.: CEPDIPO, Gentes del Común, CLACSO. 
Fundación Ideas para la Paz. (2017). Construcción de paz desde Confecámaras y las Cámaras de Comercio de Colombia. [Documento estratégico]. Bogotá, D.C.: Confecámaras y Cámara de Comercio de Bogotá. Recuperado de https://bibliotecadigital.ccb.org.co/bitstream/handle/11520/23624/Documento $\% 20 \mathrm{de} \% 20$ Acrobat.pdf?sequence $=1 \&$ isAllowed $=\mathrm{y}$

Fisas, V. (2004). Procesos de paz y negociación en conflictos armados. Barcelona: Paidós.

Galtung, J. (1995). Investigaciones teóricas, sociedad y culturas contemporáneas. Madrid: Tecnos.

Grasa, R., Carvajalino, G. y Duque, P. (2019). Construcción de paz $y$ valor compartido: Retos y oportunidades del sector empresarial en Colombia. Bogotá, D.C.: Centro de información empresarial (CEIB), Cámara de Comercio de Colombia. Recuperado de http://hdl.handle.net/11520/23385

González, F. (2014). Poder y violencia en Colombia. Bogotá, D.C.: Editorial Pontificia Universidad Javeriana.

Hernández, J., Chumaceiro H., Ziritt, G. y Acurero, M. (2018). Cultura para la paz en Colombia. Una aproximación desde las políticas públicas. Opción, 34(86), 612-641. https://produccioncientificaluz.org/index.php/opcion/article/view/23856/24300 Hernández, J., Meléndez, Y., Chumaceiro, A. y Gil, X. (2017). Cultura democrática, paz y convivencia social en Colombia. Abordaje desde una Escuela para Ciudadanos. En, J. Hernández, A. Ramírez y J. Barboza (Comp.), Conflictos y pos conflictos. Pasado y presente en América latina y el Caribe, caso Colombia, (pp. 40-58). Cabimas: UNERMB. Recuperado de http://150.185.9.18/fondo_editorial/images/ $\mathrm{PDF} / \quad \mathrm{CSF} / 00 \% 20 \mathrm{LIBRO} \% 20 \mathrm{Conflicto} \% 20 \mathrm{y} \% 20$ Postconflicto\%20\%20Hernndez\%20Ramrez\%20Barboza\%20compiladores.pdf

Ibarra-Padilla, A. M. (2014). Principios de la responsabilidad social empresarial en el ordenamiento jurídico colombiano. Revista de derecho, (41), 51-82. http://dx.doi.org/10.14482/ dere.41.5443 
Jiménez, M. C. (2016). Justicia territorial para la construcción de la paz. Bitácora, 26, 59-66. http://dx.doi.org/10.15446/bitacora.v26n2.59301

Muñoz, F. (2001). La paz imperfecta. Granada: Editorial Universidad de Granada.

Muñoz, F. y López, M. (2001). El poder pacifista. Instituto de la paz y los conflictos. Granada: Editorial Universidad de Granada.

Moreno, H. A. (2014). La paz imperfecta en el marco del conflicto armado en Colombia. Entramado, 10(1), 202-218.

Otálvaro, B. (2017). Miradas cualitativas para el análisis de políticas sociales en Colombia. Ciencia Política, 12(23), 57-78. https://doi.org/10.13446/cp.v12n23.62544

PCDHDD, CCEEU y Alianza de Organizaciones Sociales y Afines (2019). El Aprendiz del Embrujo: Finge la paz, reinventa la guerra y privatiza lo público. Balance del primer año de gobierno de Iván Duque. Bogotá, D.C.: Antropos.

Ravina-Ripoll, R., Tobar-Pesantez, L. y Marchena-Domínguez, J. (2019). A lighthouse for social wellbeing, creativity and sustainability: Happiness Management. Berna: Peter Lang.

República de Colombia. DNP. (2019). Bases del Plan Nacional de Desarrollo 2018-2022. «Pacto por Colombia, pacto por la equidad». Bogotá, D.C.

Rettberg, B. (2008). Explorando el dividendo de la paz: impactos del conflicto armado en el sector privado colombiano, resultados de una encuesta nacional. Bogotá, D.C.: Ediciones Uniandes.

Uribe, M. (2018). Infraestructuras de paz y estabilidad en Colombia. Perfiles Latinoamericanos, 26(51), 167-189. https://doi. org/ 10.18504/pl2651-007-2018

Villarraga-Sarmiento, Á. (2013). Experiencias históricas recientes de reintegración de excombatientes en Colombia. Colombia internacional, 77(1), 107-140. https://doi.org/10.7440/ colombiaint77.2013.05 
Rafael Ravina-Ripolles es Doctor en Historia y profesional en economía. Profesor asociado del departamento de Organizaciones de empresas de la Universidad de Cádiz (España). https://orcid. org/0000-0001-7007-3123

Edgar Julián Gálvez-Albarracín es Doctor en Administración y Dirección de Empresas, y Administrador de Empresas. Profesor titular de la facultad de Ciencias de la Administración y del grupo de Investigación en Humanismo y Gestión de la Universidad del Valle (Cali, Colombia). https://orcid.org/0000-0003-3972-2310

Bairon Otálvaro-Marín es Doctor en Estudios Políticos y Relaciones Internacionales. Profesor de la Facultad de Ciencias de la Administración y del Grupo de Investigación Gestión y Políticas Públicas de la Universidad del Valle (Cali, Colombia). 